\title{
Community-Based Security for the Internet of Things
}

\author{
Quanyan Zhu \\ Department of Electrical and Computer Engineering, New York University, \\ 2 Metrotech Center, Brooklyn, 11201, USA \\ Stefan Rass, Peter Schartner \\ Institute of Applied Informatics, System Security Group, Universitaet Klagenfurt, \\ Universitaetsstrasse 65-67, 9020 Klagenfurt, Austria
}

\begin{abstract}
With more and more devices becoming connectable to the internet, the number of services but also a lot of threats increases dramatically. Security is often a secondary matter behind functionality and comfort, but the problem has already been recognized. Still, with many IoT devices being deployed already, security will come step-by-step and through updates, patches and new versions of apps and IoT software. While these updates can be safely retrieved from app stores, the problems kick in via jailbroken devices and with the variety of untrusted sources arising on the internet. Since hacking is typically a community effort these days, security could be a community goal too. The challenges are manifold, and one reason for weak or absent security on IoT devices is their weak computational power. In this chapter, we discuss a community based security mechanism in which devices mutually aid each other in secure software management. We discuss game-theoretic methods of community formation and light-weight cryptographic means to accomplish authentic software deployment inside the IoT device community.

Keywords: Collaborative Systems, Smart Communities, Trust Management, Mechanism Design, Applied Cryptography
\end{abstract}

\section{Introduction}

The potential of services offered by the future internet of things is accompanied by an equally strong growing potential of new threats. The internet of things induces the trend to turn special purpose devices like TVs, radios, 
etc. into universal platforms able to execute arbitrary pieces of software and with strong communication abilities. This new power makes them vulnerable to the same types of malware that was previously only seen in classical computer networks. One of them is ransomware, which although dating back to the 1980s, sees a revival and is a major pillar of the cybercrime ecosystem today [1]. Ransomware is typically a weapon against the masses, and with the goal of pressing money. Advanced persistent threats are on the opposite being highly targeted attacks against specific victims, and typically not about monetary gain, but to demonstrate the power and to cause maximal damage. The common denominator of both extremes, and most that live between these two is their focus on the weakest element, which is typically the human. Nature itself teaches that flocks (in general communities) have much higher chances to survive than any of their individuals would have on their own. Why not adopt and systematize this well-proven behavior in security and the internet of things? Awareness of humans is a notoriously volatile state since press and media have an undoubted power in sensitizing people for a topic, but this regards every topic on which news are reported. So, more recent news tend to supersede older ones and hence awareness about threats are continuously fading away due to the mass of information that people are confronted with every day.

In that sense, the internet of things and, more generally, the information society itself creates one of the biggest dangers, by constantly overloading individuals with information so that recognizing the real danger when it occurs is harder than ever. Can we find a way out of this? There is surely no easy answer, and societal changes towards better awareness should be expected to solve the problem in near future. But human communities are not the only ones that we can create, and the internet of things, which basically is a community, offers much more controllable dynamics than any human society.

Speaking more concretely, let us look at a documented case of ransomware, having locked a TV screen [2]. The issue was apparently due to the installation of a mysterious app. More critically, the viruses are usually able to jump between different devices, partly also due to the strong prevalence of only a few platforms. For example, Android is running on tablets, mobile phones, eBook readers, TVs and many more. So, there is no technical barrier for a virulent app to pass from one device to the other. The links are exactly what IoT provides, so the world is open to any number of viruses to be fruitful and multiply and infect the world. Indeed, why not turn IoT devices them- 


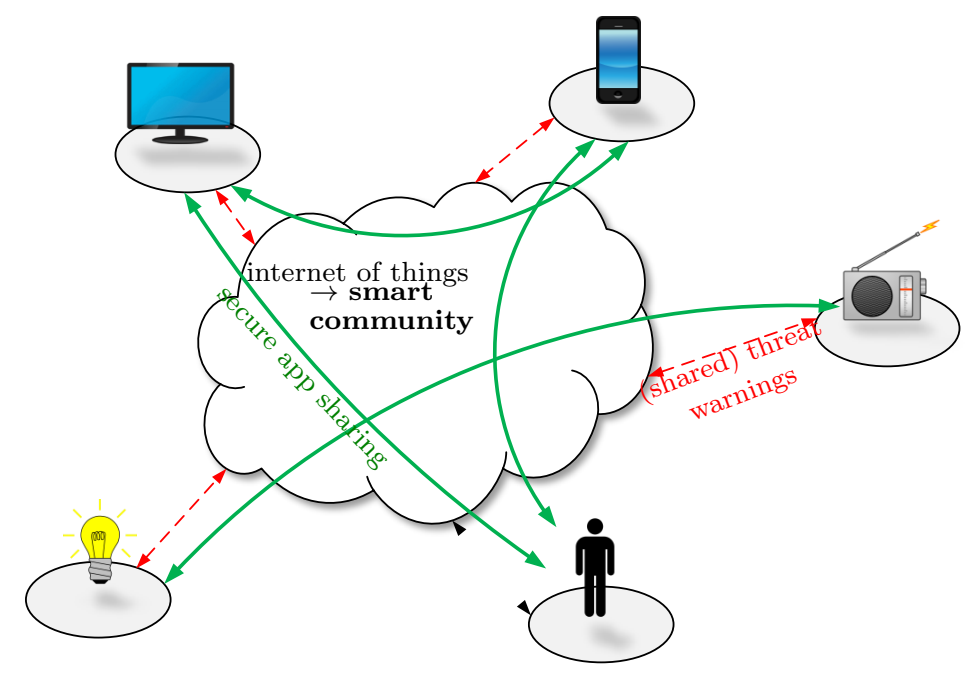

Figure 1: Turning the IoT into a Smart Community

selves into a community in which individuals (devices) mutually inform each other about threats, countermeasures and securely share apps? Since trust of humans in technology seems to be high already, why not let the awareness be up to the devices rather than the people? Figure 1 sketches the vision on this smart community living in the IoT.

With apps obtainable from various sources, only one of which is the trusted official app store, drive-by downloads have become a common way of injecting malware on mobile platforms, and perhaps also future IoT. In a drive-by download attack, the adversary obtains a legitimate copy of an app installation file (on Android, this would be an apk-file), unpacks it (as it is basically a compressed archive), adds the malware, and repacks everything back into the harmlessly looking app installation file. The unaware victim obtains the apk-file from some source and installs the app, but also the malware that unknowingly ships with it. Official key stores run by platform vendors (like Google, Apple, etc.) perform lots of screenings and security checks, and a drive-by download is most unlikely for these sources. Still, one attack vector on IoT and mobile platforms is to disconnect the user from the official app store (say, by redirecting or blocking the connection somehow), to enforce the user to look for alternative sources. There are usually many of them, but not all being equally trustworthy.

More specifically, mobile platforms can be used with mobile device man- 
agement systems (MDMs) that let anyone (primarily enterprises) run their own app stores, why not turn the community itself into a trusted app store for itself? While app stores enjoy the luxury of cryptographic security (digital signatures to verify the originality of the app), those cryptographic security precautions are not easily established inside a loose community that cannot perform a proper and decent key management.

Fortunately, there may be not even a strong need for a full-fledged public key infrastructure to endow an IoT user community with the cryptographic assurance of mutual authentication and hence trustworthiness in the shared apps! Indeed, past research has come up with proposals on how to use keys shared only with friends (or more general, the local neighborhood), to establish end-to-end authentication (and security) even with strangers with which no public key data is shared. The community, in turn, should be formed so that everyone in it has an incentive to contribute, i.e., actively communicate potential threat discoveries, and actively serve as a trusted source of data, software, and information for others. The challenge herein is building the community in the proper way to achieve (cryptographic) security for everyone. This is two problems, one of community formation, the other on security establishment. The community can be formed based on incentives so that it is the best option for everyone in the community to contribute to it. Suitable mechanisms to this end can be constructed from game theory, as we will discuss later in this chapter.

\section{State of The Art on App Security}

Taking mobile platforms as templates as to how IoT devices may look like in future, the security of mobile devices rests on four main pillars:

1. Screening of apps at app stores: before an app goes online for download, most app stores scan for malicious code or patterns (similar to what a virus scanner would do).

2. Access restrictions and permissions by the operating systems: when an app is installed, usually the required access privileges are displayed so that the user can explicitly consent. An informed decision hereby judges the combination of permissions and apps rather than each one individually, since the sum of permissions is typically much more powerful (dangerous) than each permission is on its own. At this point, care is up to the user (for example, in becoming suspicious if a torchlight 
app asks for network access; even if this app has no network access rights, it could share sensitive information via the clipboard with a "befriended" app from the same vendor that can send away the data). Newer versions of Android, for example, also allow to revoke such rights even after installation.

3. App sandboxing: within the mobile device prevents apps from mutually accessing each other's memory space or the process itself. Still, there are several possibilities to let apps talk to each other and exchange data, which can be exploited maliciously (similar to how the heartbleed exploit worked for SSL).

4. User awareness: the checks done at the app stores and everything that the operating system does hinges on the user acting carefully. This care includes an original (especially not jailbroken) copy of the platform, as well as a careful decision on which app to install. If the app's purpose is inconsistent with the privileges that it requires, then this is an indication of potential danger.

The last pillar is where the community can help the most: it is not always obvious why an app should not need some privilege, and some paid apps may have free-of-charge siblings that are simply unofficial and may thus not be found in an official app store. In both cases, community knowledge can aid and guide the user. For example, if an app is reported (to the community) as asking for strange privileges, then a warning could be issued to a new user. Likewise, untrusted sources can be replaced by the community acting as a team, thus making it more difficult for the adversary to spread its malicious content.

Using opinions is already standard in app stores, where apps get user ratings to help others find the best app for their needs. Establishing a similar system for security appears as a natural next step, and can be achieved using simple means. In fact, the cryptographic assurance that users get when retrieving an app over official channels can in a similar way be provided by (and to) the whole user community, by proper incentive and authentication mechanisms; the former resting on game theory, the latter rooting in cryptography. Hereafter, we will thus look at ways to safely distribute apps with cryptographic assurance. 


\section{Community Based Security}

The basic goal to accomplish for an attacker is tricking an unaware user into installing an app from a distrusted source. While app stores can easily certify the genuineness of their items by public key cryptography (digital signatures), not all software is obtained from these stores, and certificate management is typically a challenge of its own (despite rich and useful theory behind it).

Normally, communities rely on one or more authorities for the authentic distribution of software and threat information (warning) communication. Why not have the community do that job collaboratively? The conventional approach to authentication via public key cryptography would require a fully pervasive public key management in all devices. While this is certainly possible, the cryptographic operations are expensive (maybe too expensive for some IoT devices) and the variety of vendors will probably render the entire architecture quite complex. Symmetric cryptography, on the other hand, comes light-weight and can be used for authentication based on keys that need to be established only in the local neighborhood of a device. This simplifies matters and takes out at least one of the central authorities towards a more decentralized approach.

Also, the sharing of threat notifications and patches can be made up to the community, in addition to central such news distribution mechanisms. Essentially, the community can engage in multi-peer credibility checking towards an early warning system about drive-by downloads.

\subsection{Multi-Peer Credibility Checking}

A drive-by download occurs if an installation package is modified by an attacker to contain malicious code additionally to the actual (legitimate) app code. Adding the malware is the simple part, the tricky bit is getting someone else to download the now malicious app. One way of enforcing this is disconnecting people from the app store (temporarily), to enforce the search for alternate sources for an app.

Assume that an app has been originally distributed from a trusted source in first place, which is - in any case - the app store (hence, its role is equally crucial also in a community based security approach, as it acts as the initial point of trust before the decentralized security can come to play). Once the app - in its legitimate form - has reached some outspread, suppose a user wants to install it from a given installation package. The open source domain 
offers a simple and effective security precaution in the form of fingerprints: usually, an installation package is accompanied by several mirrors and a (cryptographic) hash-sum (often, MD5 or SHA-1). The genuineness of the downloaded file can be checked by verifying the checksum on the website. For this to work, the checksum and installation package should come from different sources, so that the confidence in the verification can be based on the unlikely event of the attacker having conquered both, the data and the checksum host(s) at the same time.

Some software systems (like the statistical software $\mathrm{R}$ or the package manager Chocolatey) by default do such a verification, which is no more expensive than one hash-and-compare operation. IoT devices will not have much difficulties in offering the necessary computational power to achieve security by the same technique; relying on the community.

Figure 2 gives an example of this process, where a user $X$ seeks to install some app on a smart TV. To get the installation package, it issues a call-out asking for others having installed the app on their (similar) devices. Suppose devices $A, B$ and $C$ have the app, while device $D$ sees the request but must remain silent since the app is not available on this device. The peers $A, B$ and $C$, however, respond by sending back a fingerprint (hash checksum) of their locally installed apps. Among the three incoming fingerprints, those from $B$ and $C$ match, while that of $A$ is different. This is already an indication that either $B$ and $C$, or $A$, must have some malicious version of the app installed. Going with a majority vote (based on the hypothesis that less infections are more likely than a pandemic outbreak of the malware), the user would thus request the app from either $B$ or $C$ (making a random choice), and inform $A$ about its suspicion to have malware there. In this way, $A$ gets informed, and can upon next possibility retrieve either a clean version of the app from the store, or communicate this fingerprint to others to check their own copies of the app (similar to a signature-based virus scan). Similar techniques are also deployed for public key management, such as for trust management in PGP and GPG [3, 4].

Once the user has found a source to get the app from, it must authentically retrieve it. This can be done by multipath authentication [5].

\subsection{Incentive Based Community Formation}

It is apparent that the credibility checking proposed relies on community building, since IoT peers need to establish a trusted neighborhood to ask for credibility and also to help with the authentication if software shall be 


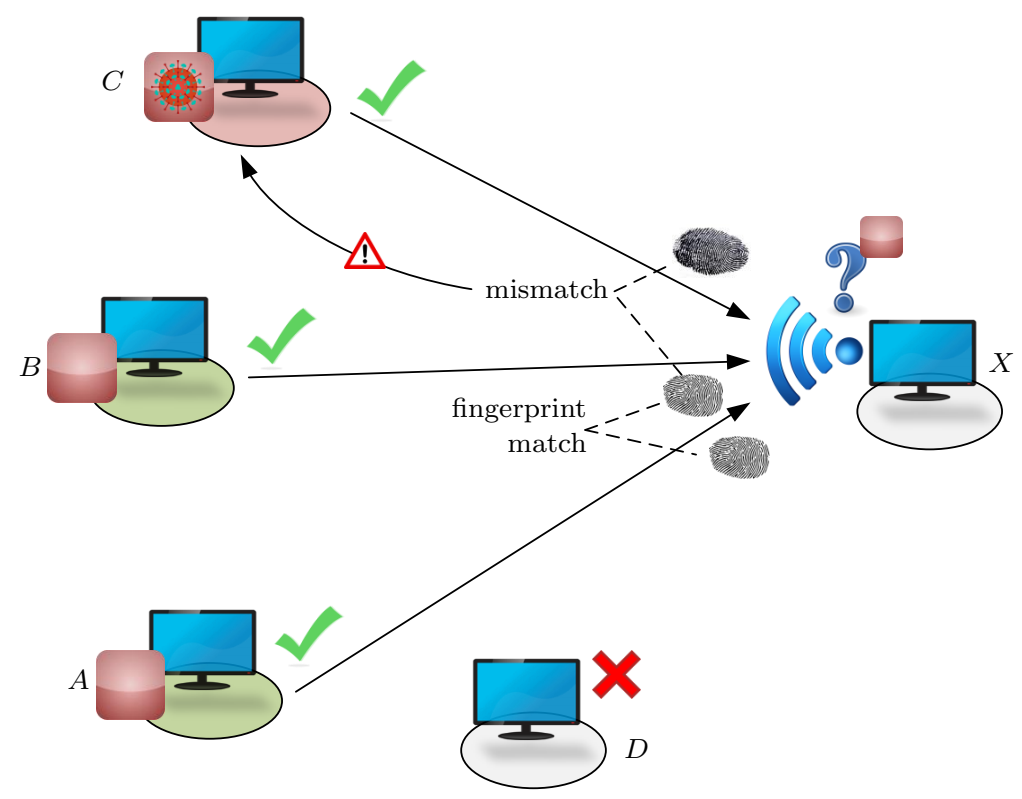

Figure 2: Multipeer Credibility Checking

deployed. Mechanisms to form these trust clusters can be understood using agent-based models and game-theoretic tools. The formation of the community is dynamic and arises from a network formation process or a cooperative game. The formation of a trustworthy IoT community plays an essential role in enabling the collaboration among devices. The community formation is naturally a dynamic problem in which nodes can join and depart the network and form communities of their interest. This process can be modeled using a network formation process in which each node is characterized by its type, preferences, trust, and utility. The type of the nodes refers to the functionalities, the age and the version of the devices. The devices of the same type often benefit more from the collaboration. However, devices of different types can also learn about emerging cyber threats and protection mechanisms from devices of similar types. Each device has a preference over the devices that it wants to communicate with. The preferences are dependent on the trustworthiness of the information received from other nodes and the utility of the collaboration. Based on the preferences, each node can choose a subset of existing nodes to initiate a request of connections and a link is formed between two nodes if the request is accepted. The acceptance of a request can be determined by a node through a cost-and-benefit evalu- 
ation; i.e., a node can determine the collaboration devices through his own preferences. This request-and-approval process is implemented at each node at every stage. As a result, a large-scale network is formed in a distributed fashion. As the parameters of the system change, nodes can terminate their collaboration with other nodes and establish new collaborations over time. In addition, new nodes will join the network and the existing nodes can leave the system. Hence the networks formed are highly dynamic. The emergence of the community of the dynamic network indicates the formation of the collaboration community. One important phenomenon that we observe is the homophily, in which nodes of the same types often form a community and share information together. Game-theoretic methods can be used to form an agent-based model to predict the structure of the network and the emergence of the communities by analyzing the Nash equilibrium of the game.

Incentive mechanism design is an important tool to create incentives in the network so that nodes actively share information with other nodes trustfully and untrustworthy devices or free-riders will be isolated and disconnected from the network. In the recent work of [6], mechanism design tools enable the system to reach a desirable and unique Nash equilibrium that allow devices to communicate in a conducive environment in which nodes endeavor to contribute knowledge and resource to assist connected nodes in the community. Any selfish or free-riding behavior will receive a tit-for-tat response from the nodes in the community as a consequence. In this way, healthy and growing collaborative communities will be achieved and maintained. Mechanism design tools can also shape the size and the structure of the network. With an appropriate design of incentive parameters, the network can grow to a desirable size by encouraging the participations from new devices. The connectivity and clustering of the community network can also be controlled by creating supernodes that behave as information hubs and incentivizing nodes to reach unconnected devices that can benefit from joining the community.

\subsection{Multipath Authentication}

Let an IoT device have made contact with its neighborhood in the network, i.e., any "surrounding" device that responds upon some sort of "HelloMessage" sent out upon the first connection (a direct connection could, for example, use a time-to-live set to 1 in order to get only the adjacent network devices; but farther distances are equally possible and legitimate here). 
Suppose that a new IoT device makes a handshake with its network neighbors, and establishes a shared secret for later authentication. The pairing can be done by any means, such as via firmware, Bluetooth, near field communication, etc. Preferably, it can be even done at manufacturing time for a whole production lot, so as to achieve a distribution in (geographic) proximity but in highly diverse networks (different homes, distinct enterprises, etc.). In (smart) homes, for example, this avoids all paired devices to communicate over the same potentially compromised hub, since the paired devices are located in different areas and under control of independent users. The mutual finding of two IoT devices can, relative to network segmentation, firewalls or other logical barriers, happen as a particular service on the application layer, which needs to be allowed to run over the IoT. However, communicating devices are the main purpose of IoT anyway.

In any case, note that unlike for general public key schemes, our schemes do not require as frequent key updates over the lifetime as IoT device. For mobile phones, as an example, an expected lifetime of 2 to 3 years (until it is replaced by a more modern version) could make a key update even unnecessary at all.

The idea of multipath authentication is the following: if $B$ wants to send an authentic message to $A$, but has neither a shared key nor public-key certificate from $A$, it employs its network neighbors to certify $B$ 's identity to $A$. To this end, $B$ shares a (distinct) key with each of its neighbors, and attaches a set of message authentication codes (MACs) to the data for $A$, indicating who $A$ should contact to have each MAC verified. Upon reception of the data and MACs, $A$ can contact each neighbor of $B$ and ask for a verification of the MACs. This validation process somewhat resembles how handwritten signatures are verified in real life upon leaving a signature sample that can be compared to the handwritten signature in question. Electronically, the process can be run just alike, at the appeal of coming cheap, since the most expensive operation is the key exchange (done only once), but all subsequent operations being fast and efficient algorithms from symmetric cryptography.

The security of the scheme, unlike that of public key cryptography, can be made independent of unproven mathematical conjectures and rests only on the assumption of a "sufficiently small" portion of the network having been compromised. In that sense, the achievable security is "unconditional". This avoids complicated assumptions that can make public key cryptography somewhat opaque to people outside the expert community (thus, adding negatively to the trust in these feelingly black boxes); see [7] for an excellent 
introduction and discussion of the issue. Finally, we remark that the way of proving security uses game-theory at the core [5].

\section{Experimental Implementations}

The concept of multipath authentication has previously been implemented and demonstrated to work on layer 7. In [8], a demonstrator has been reported that implements arbitrary secure end-to-end communication (confidential and authentic) between devices where only locally paired ones share a common secret (in an IoT setting, exchanging these is possible in various ways, such as QR codes, near-field communication, etc.). The main assumption upon which the security rests is multi-path source routing, where paths do not intersect. Given a sufficiently dense network and accurate information on the topology, it is not difficult to let the devices do the routing on the application layer (as was shown in [8], where a local Java client was used to handle these matters). Figure 3 shows a screenshot of the past prototype, which is (cryptographically) lightweight and implemented in Java to run on all platforms. The screenshot shows parts of the log of a protocol run where node 2 was asked by node 5 to verify the MACs that node 2 received from node 1 . Node 5 shares a secret with node 1 so that it can confirm (see the "boxed" part on the bottom of the window) that the MAC it computed using the secret shared with 1 matches what it received from 2 upon the authentication request. Thus, node 1's "signature" is verified by node 2 and this is told to node 5 .

\section{Proposed Architecture}

Suppose a user wants to install an app. The user has retrieved the app from somewhere (not the app store), so that the source is distrusted (typically indicated by a digital signature verification failure). What if the user

for whatever reason decides to need the app anyway? Where to get it from? Apps retrieved from the internet directly are usually not subject to the thorough screenings that app stores apply. In that case, the risk is fully taken by the user. Why not rely on community knowledge and resources in that case? The idea is the following: To retrieve the app (from elsewhere than the app store but still from a trusted source), the user (device) $X$ performs the following steps:

1. Call out to the community for "Who's got 'this app' ?" 


\begin{tabular}{|c|c|c|}
\hline \multicolumn{2}{|c|}{ RSB Demonstrator Client } & \begin{tabular}{|l|l|l}
$口$ & 回 \\
\end{tabular} \\
\hline \multicolumn{2}{|l|}{ Server IP: Iocalhost } & \multirow[t]{2}{*}{ Node ID: 2} \\
\hline Node Information & Status & \\
\hline \multicolumn{3}{|r|}{$\begin{array}{l}\text { /127.0.0.1:20001 } \\
\text { null:20005 }\end{array}$} \\
\hline \multicolumn{3}{|l|}{ Shared Keys: } \\
\hline \multicolumn{3}{|l|}{ Security Goals: } \\
\hline $\begin{array}{l}\text { Clients in Network: } \\
1: / 127.0 .0 .1: 20001 \\
5: / 127.0 .0 .1: 20005\end{array}$ & & \\
\hline \multicolumn{3}{|c|}{$\begin{array}{l}\text { 1: Received Com Packet, data: "MVJKT2FFbkxlalgySE } \\
\text { Relaying to Node } 5 \text { on remote address /127.0.0.1:20 } \\
\text { 2: Received Com Packet, data: "ems2N3M3T2IUQm5 } \\
\text { Relaying to Node } 5 \text { on remote address /127.0.0.1:20 } \\
\text { 3: Received Com Packet, data: "WmRENWxxYXVWn } \\
\text { Relaying to Node } 5 \text { on remote address /127.0.0.1:20 } \\
\text { Received Auth Packet, SHA-256 Hash: "QrqDVNsmO }\end{array}$} \\
\hline $\begin{array}{l}\text { \#\#\#\#\#\#\#\#\#\#\#\#\#\# } \\
\text { Received Mac: } \\
\text { Calculated Mac: } \\
\text { \#\#\#\#\#\#\#\#\#\#\#\#\#\#\# } \\
\text { Sending Authentific }\end{array}$ & 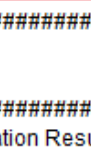 & $\begin{array}{l}\text { \# } \\
\times 2 \mathrm{HeDawNtj} \text { Uf } 2 \mathrm{Q}== \\
\mathrm{x} 2 \mathrm{HeDawNtj} \text { Uf } 2 \mathrm{Q}== \\
\text { \# } \\
\text { t to } 5 \text { on remote addre }\end{array}$ \\
\hline
\end{tabular}

Figure 3: Multipath Authentication Prototype from 8 ] 
2. Some (perhaps many) devices may respond by sending a confirmation to have the app and sending the fingerprint (hash of the app file), upon which a set of $h_{1}, \ldots, h_{n}$ arrives at the user. Devices that are too old should be abandoned from that list e.g. if their key length (for calculating the MACs in step 5 is less than the current recommendations.

3. The user $X$ goes for a majority vote and uses the hash that appears the most among $h_{1}, \ldots, h_{n}$, following the hypothesis that the malware has not yet affected too large parts in the community. Let $h_{i}$ be that majority value received from the $i$-th respondent. Let the user having sent $h_{i}$ be called $B$ (as in Figure 4).

4. If any of the hashes mismatches another, then we have an indication of some malware potentially being around in the community.

5. To retrieve the app, $X$ contacts $B$ to send the app with fingerprint $h_{i}$ as known from step 4 . User $B$ then runs multipath authentication to send an authentic version of the app. In Figure 4, $B$ attaches MACs using the keys shared with its neighbors and sends the app file with the MACs to $X$. This one compares the fingerprint of the received app to match what it should be (namely $h_{i}$ ), and asks then (indicated) neighbors of $B$ to verify the hash of the app and the MAC attached to it. This prevents $B$ from sending a correct fingerprint but a malicious version of the app later (since the fingerprints would mismatch then), and assures $X$ the authenticity of $B$ 's app packet.

The plain protocol can be adapted towards a less stringent yet no less informed behavior, in case that users are willing to accept a certain residual risk in the retrieval process. If so, then quantifying that risk is the major objective, and done as follows:

Let $N=\{1,2,3, \ldots\}$ represent the community, with physical member IDs being numbers. To each member $i \in N$ of the community, we associate a trust value based on the following intuition: if, in the above protocol, a user $X$ may query another user $B$ asking to respond to a MAC verification. Ultimately, the user $X$ is interested in the trustworthiness of the retrieved app; let us call this event $T \in\{0,1\}$, where $T=0$ is zero trust and $T=1$ is full trust. Ultimately, we are interested in the distribution of $T$ over the unit interval $[0,1]$, conditional on the information available to $X$, i.e., our trust measure is $\tau=\operatorname{Pr}$ (app is trustworthy|user $i$ says so), or more compactly, $\tau=\operatorname{Pr}(T=1 \mid R)$. Note that $X$ polls only a subset of app sources/users $u_{1}, \ldots, u_{n} \in N$, so that the probability space in which $T$ lives will not be 
partitioned by the events $R_{i}$ associated with the peers in the protocol. To fix this, we replace $T$ by $T_{X, B}$, as being the subjective trust level that user $X$ assigns to the app retrieved from user $B$, based on the information available. This (conditional) random variable has its probability space partitioned by the users that $X$ contacts, but the fix comes with the caveat of the objective trust $T$ remaining out of reach, with only the subjective trust $T_{X, B}$ assigned by $X$ being computable. The distribution of $T_{X, B}$, however, follows from the law of total probability,

$$
\operatorname{Pr}\left(T_{X, B}\right)=\sum_{i=1}^{n} \operatorname{Pr}\left(T_{X, B}=1 \mid R_{i}\right) \operatorname{Pr}\left(R_{i}\right) .
$$

Mechanism design is herein concerned with the question of why a user should be willing to honestly participate in the protocol. The incentive for user $i$ to respond correctly, by (1) is positively correlated to the subjective trust that user $X$ obtains from asking user $i$. Since $i$ is, by construction, $B$ 's neighbor, whatever $i$ reports back depends on the trust that $i$ has in $B$. But the situation is symmetric: $i$ has an incentive to answer faithfully about $B$, since whichever information or app is ever retrieved from $i, B$ is perhaps among the neighbors to be queried, so if $i$ refrains from responding or responds incorrectly, it may indirectly damages its own reputation in the long run. The same symmetry, however, cannot straightforwardly be used by a malicious $i$ to damage $B$ 's reputation, since there is still the set of other neighbors that may indicates $B$ 's honesty (recall that the protocol goes for the majority vote among all the replies).

Practically, we have a triple of trust values, which $X$ maintains about the community:

1. The likelihood $\operatorname{Pr}\left(R_{i}\right)$ that user $i$ responds. Based on whether or not $i$ participated in the above protocol, $X$ can update the trust value for $i$ accordingly. If $i$ refuses to reply about $B, B$ can later refuse to tell about $i$, which is $i$ 's indirect incentive to become active.

2. The likelihood $\operatorname{Pr}\left(T_{X, B}=1 \mid R_{i}\right)$ that user $i$ 's reply was correct and helpful. This value is updated upon the outcome of the majority vote made along the protocol, as discussed before.

3. The trust value $\operatorname{Pr}\left(T_{X, B}=1\right)$ computed from (1), based on the previous two items.

This form of app retrieval is designed towards simplicity and security at 


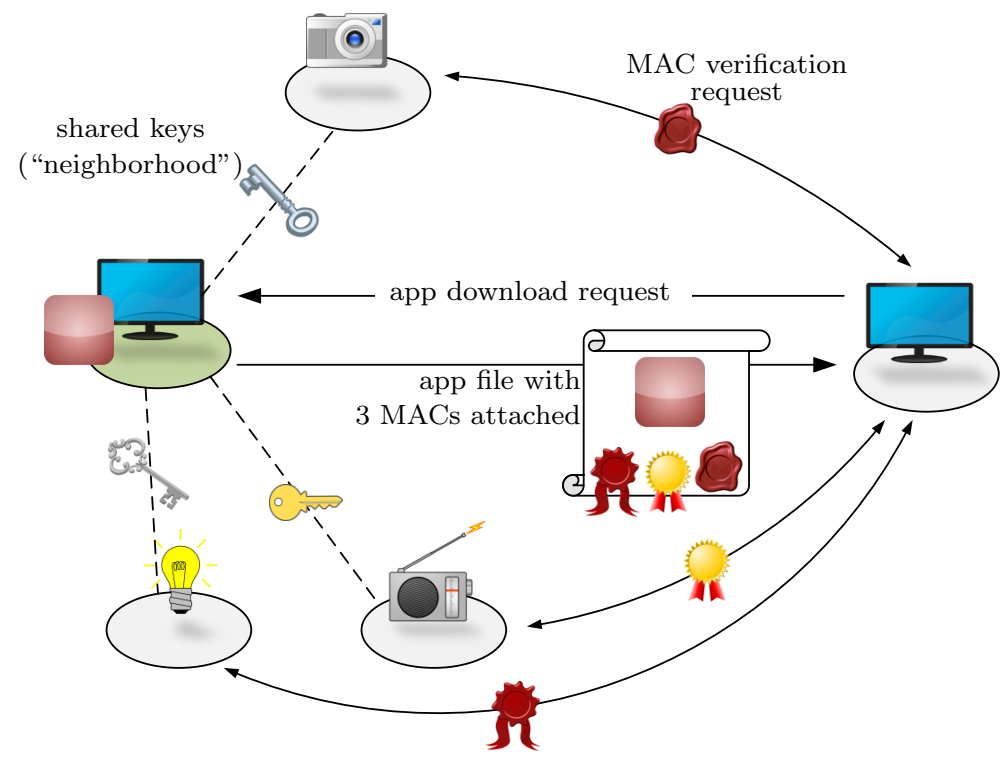

Figure 4: Multipath Authentication

the same time: note that the community - by collecting and comparing app fingerprints - continuously builds up some sort of threat intelligence. Note that the hash in step 1 and the MAC in step 5 cryptographically link the app discovery and the app download. Otherwise, a malicious device may present one (harmless) app at step 1 and another (malicious) one at step 5 (cf. time of check to time of use - TOCTTOU [9]).

The bandwidth increase is the primary price to pay for this scheme to work, but remember that all we need to exchange are cryptographic hash values, which are only a few bytes long. For a quick calculation: using a 224 Bit SHA-3 checksum (state of the art) and with 10 peers contacted, the following bandwidth is required: we have $10 \times 224$ bit $\approx 2 \mathrm{kBit}$ for the initial call out. Now, if the app sender upon request uses 10 of its neighbors to verify its authenticity, we end up with a total of $2 \mathrm{kBit}$ for the $10 \mathrm{MACs}$, and another $10 \times 2 \times 224$ bit $\approx 4.5 \mathrm{kBit}$ for all verifications. Thus, a total of less than $10 \mathrm{kBit}$ of overhead is required for 10 neighbors (more or less peers induce a proportional increase or decrease respectively).

We finally stress that this entire scheme can be constructed on Layer 7, i.e., no deep changes to the devices or their network stack is necessary. In fact, a demonstration prototype of multipath authentication (in the natural 
combination with multipath transmission) has been successful already.

The local management of keys is herein aligned with the app sandboxing implemented on all commercial mobile phone platforms. General IoT devices are expected to run the same (or at least similar) operating systems. Platforms like Android enforce sandboxing towards preventing apps from accessing the memory blocks assigned to other apps. Thus, the cryptographic keys are essentially locally safe by logic access control by the operating system.

\section{Outlook}

The system discussed in this article already has close relatives up and running to warn users about malicious websites or changed/outdated public key certificates [10, 11, 12]. Provided that such technology does not itself exhibit unwanted behavior otherwise (like unauthorized data collection), why not use similar technology in the IoT?

Hacking and threat intelligence are community actions, and so should security and mutual protection be. Large (animal) populations protect themselves by forming herds and IoT devices can do similar things to harden the whole community against external threats. We believe that a comprehensive security concept should not exclusively rest on cryptographic mechanisms, but should to a wide extent include incentive and credibility-driven mechanisms to let users (devices) collaborate to the good of everyone. A combination of mechanism design [13, game theory and cryptography can make a start here, but the diversity of the IoT seems to call for a scientific treatment with tools that are equally diverse.

\section{References}

\section{References}

[1] E. Maor, Cybercrime ecosystem: Everything is for sale, [retrieved: November 15, 2017] (2015).

URL https://securityintelligence.com/ cybercrime-ecosystem-everything-is-for-sale/

[2] C. Cimpanu, Android ransomware infects lg smart tv, [retrieved: November 15, 2017] (2016). 
URL https://www.bleepingcomputer.com/news/security/ android-ransomware-infects-lg-smart-tv/

[3] J. Callas, H. Donnerhacke, H. Finney, D. Shaw, R. Thayer, Openpgp message format, [retrieved: November 15, 2017] (2007).

URL https://tools.ietf .org/html/rfc4880

[4] P. Penning, Henk, Analysis of the strong set in the PGP web of trust, [retrieved: November 15, 2017] (2016). URL https://pgp.cs.uu.nl/plot/

[5] S. Rass, P. Schartner, Multipath authentication without shared secrets and with applications in quantum networks, in: Proceedings of the International Conference on Security and Management (SAM), Vol. 1, CSREA Press, 2010, pp. 111-115.

[6] Q. Zhu, C. Fung, R. Boutaba, T. Basar, Guidex: A game-theoretic incentive-based mechanism for intrusion detection networks, Selected Areas in Communications, IEEE Journal on 30 (11) (2012) 2220-2230. doi:10.1109/JSAC.2012.121214.

[7] N. Koblitz, A. J. Menezes, Another look at "provable security", J. Cryptol. 20 (1) (2007) 3-37. doi:10.1007/s00145-005-0432-z.

[8] S. Rass, B. Rainer, M. Vavti, J. Göllner, A. Peer, S. Schauer, Secure communication over software-defined networks, Mobile Networks and Applications 20 (1) (2015) 105-110. doi:10.1007/s11036-015-0582-7.

[9] C. Mulliner, B. Michéle, Read it twice! a mass-storage-based TOCTTOU attack, in: Presented as part of the 6th USENIX Workshop on Offensive Technologies, USENIX, Bellevue, WA, 2012.

URL https://www.usenix.org/conference/woot12/ workshop-program/presentation/Mulliner

[10] D. Wendlandt, D. Anderson, A. Perrig, Perspectives project (firefox browser add-on), [retrieved: November 15, 2017] (2015). URL https://perspectivessecurity.wordpress.com/

[11] WOT Services, Mywot/wot: Website reputation rating (firefox browser add-on), [retrieved: November 15, 2017] (2015). 
URL https://addons.mozilla.org/EN-us/firefox/addon/ wot-safe-browsing-tool/

[12] C. von Loesch, Certificate Patrol, v.2.0.16 (firefox browser add-on), [retrieved: November 15, 2017] (2015).

URL https://addons.mozilla.org/de/firefox/addon/ certificate-patrol

[13] T. Börgers, D. Krähmer, R. Strausz, An introduction to the theory of mechanism design, Oxford University Press, Oxford, 2015. 